\title{
Active Disturbance Rejection Vibration Control for an All-clamped Piezoelectric Plate with Delay
}

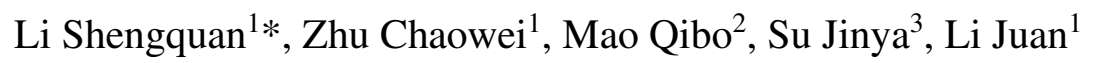 \\ 1, College of Electrical, Energy and Power Engineering, Yangzhou University, \\ Yangzhou 225127, China \\ 2, School of Aircraft Engineering, Nanchang HangKong University, Nanchang, China \\ 3, School of Computer Science and Electronic Engineering, University of Essex, \\ Colchester CO4 3SQ, U.K.
}

\begin{abstract}
All-clamped plate structures are usually subject to strong coupling, model uncertainties and system time-delay. To address these challenges, this work proposes a novel vibration control method based on a linear active disturbance rejection controller (LADRC) with time-delay compensation (TDC-LADRC). The mathematical model of the piezoelectric plate is first established based on system identification with an auxiliary variable method. Then ADRC is designed for the delay-free part by a smith predictor with a novel differentiator. An extended state observer (ESO) is drawn to estimate the internal and external disturbances, such as mode errors, higher harmonics and external environmental excitations. Then, real-time compensation is introduced via feed-forward mechanism to attenuate their adverse effects, so that optimal vibration suppression performance can be achieved by the proposed controller. Finally, based on NI-PCIe6343 acquisition card, an experimental set-up is designed to verify and compare the performance of the proposed TDC-LADRC against the traditional LADRC and the traditional predictor based LADRC (PLADRC). Comparative experimental results show that the proposed TDCLADRC possesses the best disturbance rejection and vibration suppression performance.
\end{abstract}

Keywords: all-clamped piezoelectric plate; linear active disturbance rejection control (LADRC); extended state observer (ESO); active vibration suppression; system delay.

*Corresponding Author. Tel.: +86 514-87971315; Email address: sqli@ yzu.edu.cn 


\section{Introduction}

An all-clamped plate is a thin structure with many excellent features, such as simple structure, light weight and high flexibility, and therefore, it has been widely applied in practical industries, i.e., automobile, shipbuilding, aeronautic and aerospace engineering ${ }^{[1,2]}$. Serious vibrations can be easily caused by internal and external disturbances in practical applications, which will reduce system reliability and comfortableness. In addition, the long-term and large-scale vibration of the thin plate structure may result in fatigue crack and structural damages, or even result in-heavy potential accidents in some extreme situations. Thus, the suppression of the destructive vibration therein has great application significances in industrial fields. With recent development of the piezoelectric structure, the piezoelectric elements can be embedded into the body thin plate structure as the-sensors or the-actuators. As a result, this type of smart piezoelectric elements has become attractive alternative materials for structural vibration control due to their excellent mechanical-electrical coupling, frequency response and reliability ${ }^{[3]}$.

In order to actively control the vibration of the actual all-clamped thin plate with piezoelectric elements, different control methods have been proposed to address the challenges caused by uncertain dynamics, complex boundary conditions, various disturbances_-(e.g. external excitations), electromechanical coupling characteristics, structure nonlinearity, and saturation of the sensing and actuating capability ${ }^{[4,5]}$. Thus, it is paramount to design active vibration control strategies for this kind of piezoelectric structures with satisfactory vibration suppression performance ${ }^{[6-25]}$.

Traditional feedback-based active vibration control methods are the natural choices and have been widely applied in the piezoelectric structural vibration suppression, such as optimal control strategies ${ }^{[10]}$, internal model controller (IMC) ${ }^{[11]}$, 
PID-based control methods ${ }^{[12-14]}$, pole assignment schemes ${ }^{[15]}$, velocity negative feedback controller ${ }^{[16,17]}$, robust control methods ${ }^{[18,19]}$, positive position feedback (PPF) controller ${ }^{[20,21]}$, model predictive controller (MPC) ${ }^{[22,23]}$, sliding model control (SMC) method ${ }^{[24,25]}$. These methods, especially the optimal linear quadratic regulator/ Gaussian (LQR/LQG), pole assignment method, PPF and negative feedback controller, have a simple frame-structure and can be easily implemented. The aforementioned vibration control methods have their pros and cons and can improve the whole vibration suppression performance in some aspects. However, an accurate mathematical model for the all-clamped piezoelectric plate is generally unavailable due to boundary condition complexities and system uncertainties. It is therefore difficult to obtain satisfying vibration suppression performance by these methods, since they cannot reject the internal and external disturbances directly and effectively [26]. The MPC and SMC methods are the exceptions due to their strong anti-disturbance ability. However, it is noted that the chattering phenomenon is inevitable in traditional SMC methods for practical structural vibration suppression, which restricts its practical applications ${ }^{[25,27]}$. MPC can determine the optimal control value through an online optimization process with physical boundary constraints, since this method employs the dynamic model of the plant to estimate its future behavior at each sampling time. However, this method will degrade significantly in the presence of strong internal and external disturbances, since the performance of MPC depends on the an accurate system and disturbance models ${ }^{[28]}$. Therefore, the structural vibration controller for piezoelectric plate should be designed rationally, considering the complexity of electromechanical vibration model caused by system disturbances, uncertainties and complex boundary conditions. 
The increasing requirements of structural vibration suppression have motivated researchers to further improve the feedback-based controllers en-by designing the controller directly and efficiently against the disturbances ${ }^{[3,4]}$. The feed-forward compensation for internal and external disturbances is considered as an ideal way, in addition to the conventional feedback part, resulting in a composite controller. However, the conventional feed-forward-based vibration controllers are commonly infeasible in many practical structural vibration suppression applications, since the mechanism models or measurements of the disturbances are generally unavailable. To this end, several disturbance estimation methods, such as extended state observer (ESO), disturbance observer (DOB), perturbation observer (PO), equivalent input disturbance (EID), generalized proportional integral observer (GPIO), uncertainty and disturbance estimator (UDE) and unknown input observer (UIO), have been proposed as alternatives to design such composite vibration control methods ${ }^{[29-47]}$. Many researchers have presented a variety of practical and effective vibration control techniques based on these disturbance estimation methods. The DOB based vibration control can estimate the disturbances by using system input, output and model information, which can be used to compensate for the adverse effects of the disturbances ${ }^{[30-34]}$. The PO based vibration control is an active anti-disturbance technology developed on the basis of DOB method. This approach acts as an internal model controller to estimate and compensate the structural internal and external disturbances through a feed-forward channel ${ }^{[35,36]}$. The EID based vibration control attenuates the disturbances with an equivalent form based on system input and output without rank conditions or disturbance and they havehas an excellent anti-disturbance ability and stability ${ }^{[37-39]}$. The GPIO-based vibration control method can simultaneously estimate unknown state variables of the structural vibration modes, 
disturbances and their derivatives ${ }^{[40-42]}$. It can suppress the structural vibrations caused by sinusoids, constants and parabolas disturbances, and can be easily combined with other advanced feedback control methods. Considering the model uncertainties, UDE-based vibration control method can estimate and compensate the internal and external disturbances by introducing an appropriate filter in frequency domain ${ }^{[43-45]}$. The disturbances can be mainly estimated by using the system state variables to improve the dynamic and static vibration suppression performance of the whole system in UIO-based vibration control methods ${ }^{[46,47]}$.

ESO is another disturbance estimation concept originally proposed by Han in 1995 and fully presented in 2009. ESO-based control method, also named active disturbance rejection controller (ADRC), regards the internal and external disturbances as an extended system state variable without the-a detailed mathematical model of the structural vibration system. The disturbances may include uncertain excitations, coupling effects, modeling errors, nonlinearities and parametric variations. Due to its strong anti-disturbances ability, fewer tuning parameters and a simple structure $_{2}$ linear ADRC (LADRC) has been applied to the complex structural vibration systems in recent years ${ }^{[48-52]}$.

Besides these internal and external disturbances, time delay is also a significant issue in ADRC methods ${ }^{[53-56]}$. The time delay of the structural vibration control system may arise from many factors such as computational delay, time of signal passing through power amplifier, and the-sampling time of the data acquisition card, etc. Additionally, the phase lag, due to the inevitable non-collocated placement of the acceleration sensor and piezoelectric actuator, is also an important constitutive factors of system time delay. Thus, time delay is also a big challenge in structural vibration system. A smith predictor technology based LADRC (PLADRC) vibration control 
method is proposed to compensate time delay caused by the non-collocated placement of the sensor/actuator pair in [49]. The experimental results show that the PLADC can effectively suppress the structural vibration. It should be noted that smith predictor based vibration control involves acceleration differentiation, and the actual vibration sensing signal always contains a high frequency noise in-a certain frequency bands. As a result, PLADRC vibration controller may degrade the suppression performance due to high frequency noise amplification.

Considering the characteristics of the all-clamped piezoelectric thin plate, this paper aims to deal with the critical issue in structural vibration control system: the rejection of internal and external disturbances. In particular, LADRC based vibration controller is proposed to suppress the modal vibration of piezoelectric smart structure with complex boundary conditions. In addition, to attenuate the adverse effect of time delay, the smith predictor can be transformed by system output and its differentiation with an enhanced differentiator. Since the ADRC vibration control algorithm can act on the time-delay-free link, it can achieve the optimal suppression of structural vibration.

In this paper, a LADRC-based structural vibration controller is designed to enhance the vibration suppression performance of an all-clamped piezoelectric plate structure. The main contributions of the proposed vibration control strategy are summarized as follow: (1) It establishes a practical electromechanical model for the piezoelectric plate structure by combining system identification and auxiliary variable method; (2) It achieves excellent vibration suppression performance and superior robustness against strong couplings, model uncertainties and external excitations; (3) It introduces a novel differentiator based smith predictor with Lissajous curves method into the vibration control loop, which enables a more efficient ESO for system delay; 
(4) It designs a hardware-in-the-loop system based on a NI data acquisition system to verify the effectiveness and superiority of the proposed active vibration controller. The remainder of this paper is organized as follows. Mathematical model of an all-clamped piezoelectric smart plate is described in Section 2. In Section 3, the design of a composite vibration controller for the piezoelectric plate is detailed. Moreover, the superiority of the improved differentiator is analyzed and verified by a vibration signal with a main frequency of $51.9 \mathrm{~Hz}$. In Section 4, the hardware-inthe-loop system of an all-clamped piezoelectric thin plate for active vibration control is designed and built up based on NI-PCIe6343 acquisition card and the real-time module of Matlab/Simulink. The Ttime delay coefficient of the whole system is experimentally identified by excitation analysis. Experimental results are presented to compare the proposed time delay compensation ADRC (TDCLADRC) against the traditional LADRC and PLADRC. Finally, Section 5 concludes the work.

\section{Mathematical Model of an All-clamped Piezoelectric Thin Plate}

An all-clamped thin plate structure is made of aluminium alloy equipped with piezoelectric element as actuator and accelerometer as sensor. The acceleration sensor is arranged close to the piezoelectric bimorph actuator to avoid non-collocated sensor/actuator pairs. Additionally, the sensor and actuator pairs are very light in this paper, and therefore their effects are very slightmarginal in modeling the all-clamped piezoelectric plate structure. Although the engineering plate structure is continuous

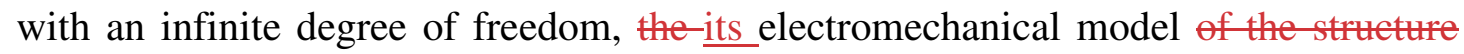
can be described as a second order mass-damper-stiffness system_, when the structureit is excited near a certain modal frequency. In addition, the whole structure with piezoelectric elements assumes a linear elasticity. So, the whole dynamic equation of the all-clamped piezoelectric plate in Fig. 1 can be described by following 
Eq. (1), similar as refs. [48, 49]:

$$
M \ddot{d}(t)+C \dot{d}(t)+K d(t)=F(t)+B V(t),
$$

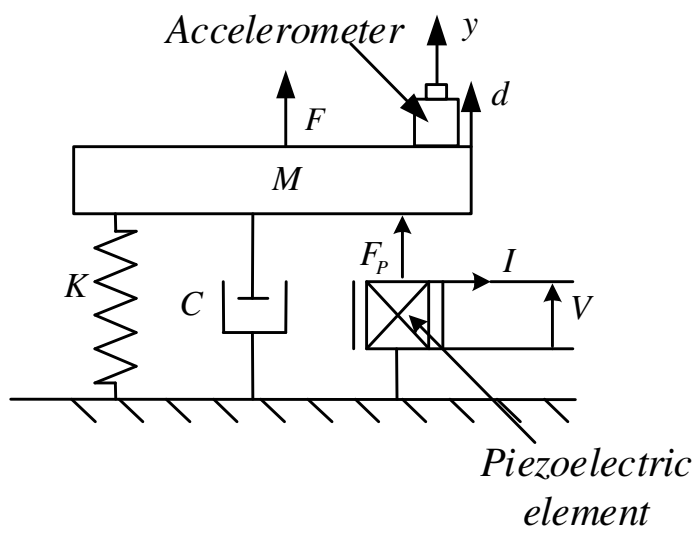

Fig. 1 Diagram of an all-clamped piezoelectric thin plate structure

where the modal parameters $M, C, K, d, y$ are the mass, damping, stiffness, displacement and acceleration, respectively. The parameter $F$ represents the sum of other forces applied to the equivalent rigid mass, including the external excitation. The parameters $B, I$ and $V$ are defined as force factor, outgoing current and input voltage of piezoelectric bimorph, respectively. The control effect of piezoelectric actuator is represented by symbol $F_{P}$ which is equal to $B$ times $V$. Considering the complexity of the piezoelectric structure, the-various parameters cannot be simply obtained from the properties of the piezoelectric inserts. The model parameters $M, C$ and $K$ can also be identified by the model test method ${ }^{[31,48,49]}$. The test values of the three model parameters are related to the short circuit frequency and the open circuit frequency of the piezoelectric patches. However, the measurement accuracy of these two frequencies are affected by the arrangement of piezoelectric elements and the temperature of the epoxy resin layer for pasting.

Another common modelling method is system identification with system 
behaviors information provided byvia system input and output. Considering the system characteristics-of the second order system of a certain model structurat vibration, an anti-colored noise based auxiliary variable method is employed to model the structural vibration system which consists of all-clamped plate, piezoelectric actuator, power amplifier, acceleration sensor, data acquisition card and processor. Similar as ref. [57], a linear discrete-time single-input-single output (SISO) system can be considered by following Eq. (2):

$$
a_{y}\left(z^{-1}\right) y(k)=b_{u}\left(z^{-1}\right) u\left(k-d_{t}\right)+\eta(k)
$$

where $z^{-1}$ and $d_{t}$ are the delay factor and system delay, respectively. Parameter $\eta(k)$ represents the system noises. Functions $a_{y}(\cdot)$ and $b_{u}(\cdot)$ are the input and output polynomials. In addition, the following sets of vectors are defined for simplicity.

$$
\begin{aligned}
& \mathbf{Q}=\left[a_{1}, a_{2}, \cdots, a_{n a}, b_{1}, b_{2}, \cdots, b_{n b}\right]^{T}, \\
& \mathbf{H}(k)=[-y(k-1), \cdots,-y(k-n a), u(k-d-1), \cdots, u(k-d-n b)]^{T}, \\
& \mathbf{Y}_{L}=[y(1), y(2), \cdots, y(L)]^{T}, \\
& \mathbf{\eta}_{L}=[\eta(1), \eta(2), \cdots, \eta(L)]^{T}, \\
& \mathbf{H}_{L}=[h(1), h(2), \cdots, h(L)]^{T},
\end{aligned}
$$

where subscript $n a$ and $n b$ are the orders of polynomials $a_{y}(\cdot)$ and $b_{u}(\cdot)$, respectively. The parameter $L$ represents the system length. The system model of Eq. (2) can be described in the following least square form:

$$
\mathbf{Y}_{L}=\mathbf{H}_{L} \boldsymbol{\theta}+\boldsymbol{\eta}_{L}
$$

where $\boldsymbol{\theta}$ represents the undetermined system parameter matrix, which can be 
estimated by the following least squares method.

$$
\boldsymbol{\theta}_{L S}=\left(\mathbf{H}_{L}{ }^{T} \mathbf{H}_{L}\right)^{-1} \mathbf{H}_{L}{ }^{T} \mathbf{Y}_{L}
$$

Considering the noise of acquisition system, $\boldsymbol{\eta}_{L}$ is a colored noise, so the parameter $\boldsymbol{\theta}_{L S}$ is not the unbiased uniform estimated values. The dimension of auxiliary variable matrix $\mathbf{H}_{L}^{*}$ is $(n a+n b) \times L$. Considering equations $\lim _{L \rightarrow \infty} \frac{1}{L} \mathbf{H}_{L}{ }^{* T} \boldsymbol{\eta}_{L}=0$ and $\lim _{L \rightarrow \infty} \frac{1}{L} \mathbf{H}_{L}{ }^{* T} \mathbf{H}_{L}=\mathbf{Q}$ with a nonsingular matrix $\mathbf{Q}$, the unbiased uniform estimation values of parameter matrix can be described as follows

$$
\tilde{\boldsymbol{\theta}}_{I V}=\left(\mathbf{H}_{L}{ }^{* T} \mathbf{H}_{L}\right)^{-1} \mathbf{H}_{L}^{{ }^{*} T} \mathbf{Y}_{L}
$$

Considering the characteristics of the structural modal vibration, the all-clamped thin plate equipped with piezoelectric bimorph in Fig. 1 is a typical second-order electromechanical system. The transfer function of the all-clamped piezoelectric thin plate in Eq. (6) can be obtained by the above mentioned auxiliary variable identification method. The parameters of Eqs. (2) to (5) can be measured by the experimental setup in Section 4.

$$
Q(s)=\frac{y(s)}{u(s)}=\frac{k_{0}}{s^{2}+k_{1} s+k_{2}} e^{-\tau s},
$$

where $k_{0}, k_{1}$ and $k_{2}$ are the model parameters obtained by the parameter matrix. The time constant $\tau$ is the time delay parameter estimated by the Lissajous method.

\section{Composite Vibration Controller Design}

\subsection{Design of an Enhanced Smith Predictor}

The system transfer function Eq. (6) consists of two parts including the time delay and nominal model. The control value will lag behind the measurement output signal, so the vibration suppression performance will be degraded or even instability occurs. 
Before designing ADRC vibration controller, the model vibration part can be converted into a delay-free-part system by introducing Smith Predictor in Fig. 2.

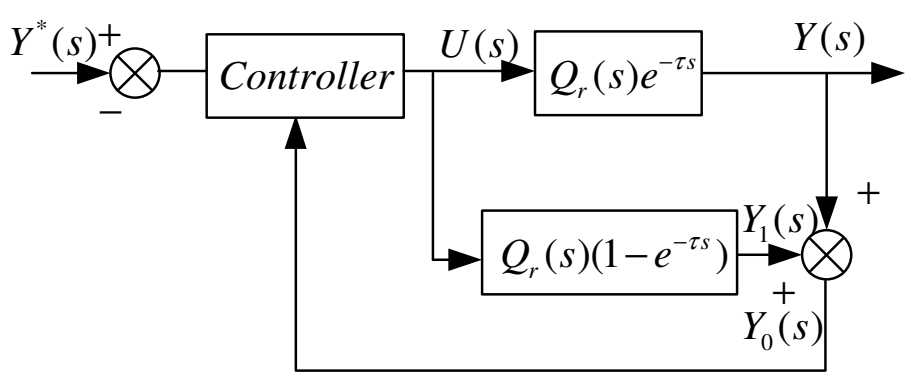

Fig. 2 Schematic diagram of the Smith Predictor

According to the substance of the Smith Predictor method and the features of the all-clamped piezoelectric structural vibration system Eq. (6), the transfer function of whole system in Fig. 2 can be described by Eq. (7).

$$
Q(s)=Q_{r}(s) e^{-\tau s},
$$

where $Q(s)$ and $Q_{r}(s)$ represent the actual and normal system model, respectively. From Fig. 2, an auxiliary output variable $Y_{0}(s)$, defined in Eq. (8), is introduced to offset the effect of time delay part.

$$
Y_{0}(s)=Y(s)+Y_{1}(s)=Q_{r}(s) e^{-\tau s} U(s)+Q_{r}(s)\left(1-e^{-\tau s}\right) U(s)=Q_{r}(s) U(s) .
$$

The transfer function between the input $u$ and the auxiliary output variables $Y_{0}(s)$ does not contain time delay part, according to Eq. (8). In addition, the output value of smith predictor $Y_{1}(s)$ is hard to be directly obtained in actual experiments. One has $e^{\tau s} \approx 1+\tau s$, when the time delay $\tau$ is a small constant. Then Eq. (8) can be further rewritten as follows: 


$$
\begin{aligned}
Y_{0}(s) & =Q_{r}(s) U(s)=Q_{r}(s) e^{-\tau s} U(s) e^{\tau s} \approx Y(s)(1+\tau s) . \\
& =Y(s)+\tau Y(s) s
\end{aligned} .
$$

The corresponding time domain expression of Eq. (9) is satisfiedsatisfied that $y_{0}(t)=y(t)+\tau y(t)$, where $y_{0}(t)$ and $y(t)$ are the time domain form of $Y_{0}(s)$ and $Y(s)$. So the auxiliary output $y_{0}(t)$ can be obtained by system output $y(t)$ and its derivatives. In engineering applications, the traditional differentiator is realized by the transfer function $\omega(s)=\frac{s}{\tau s+1}$. The function of $d u / d t$ in Matlab/Simulink system is also achieved by this method. The differentiation of output $y$ can be obtained approximately by Eq. (10):

$$
\begin{aligned}
s Y(s) & =\omega(s) Y(s)=\frac{s}{\tau s+1} Y(s)=\frac{1}{\tau}\left(1-\frac{1}{\tau s+1}\right) Y(s) \\
& =\frac{1}{\tau}\left(Y(s)-\frac{1}{\tau s+1} Y(s)\right)
\end{aligned},
$$

where $1 /(\tau s+1)$ is an inertial transfer function of time constant $\tau$. One has $Y(s) /(\tau s+1) \approx Y(s) e^{-\tau s}$ when the output signal $Y(s)$ changes slowly. So, the output differentiation Eq. (10) can be further obtained by Eq. (11).

$$
\dot{y}(t) \approx \frac{1}{\tau}(y(t)-y(t-\tau)) .
$$

In the actual vibration signal processing, the acceleration sensor not only collects the system vibration, but also noises from the external environment. Considering the signal output $y$ with noise $\eta$, the actual derivative $\dot{\hat{y}}$ of $d u / d t$ can be described as follows: 


$$
\begin{aligned}
\dot{\hat{y}}(t) & =\frac{1}{\tau}(y(t)+\eta(t)-y(t-\tau)) \\
& \approx \dot{y}(t)+\frac{1}{\tau} \eta(t)
\end{aligned}
$$

Eq. (12) indicates that the noise $\eta$ can be amplified by the time delay constant $1 / \tau$ in differentiation. In order to attenuate the influence of the noise mixed in actual vibration signal, the following differential approximation equation (13) is proposed to enhance purity of the acquisition vibration signal.

$$
\dot{y}(t) \approx \frac{y\left(t-\tau_{1}\right)-y\left(t-\tau_{2}\right)}{\tau_{2}-\tau_{1}}, 0<\tau_{1}<\tau_{2} .
$$

The delay signals $y\left(t-\tau_{1}\right)$ and $y\left(t-\tau_{2}\right)$ can be approximately replaced by the first-order inertial transfer functions $1 /\left(\tau_{1} s+1\right)$ and $1 /\left(\tau_{2} s+1\right)$, respectively. So the enhanced differential equation in Fig. 3 can be obtained by Eq. (14):

$$
\begin{aligned}
s Y(s) & =\frac{1}{\tau_{2}-\tau_{1}}\left(\frac{1}{\tau_{1} s+1}-\frac{1}{\tau_{2} s+1}\right) Y(s) \\
& =\frac{s}{\tau_{1} \tau_{2} s^{2}+\left(\tau_{1}+\tau_{2}\right) s+1} Y(s)=\omega^{*}(s) Y(s) \\
\longrightarrow \frac{1}{\tau_{1} s+1} & \longrightarrow \frac{1}{\tau_{2} s+1}
\end{aligned}
$$

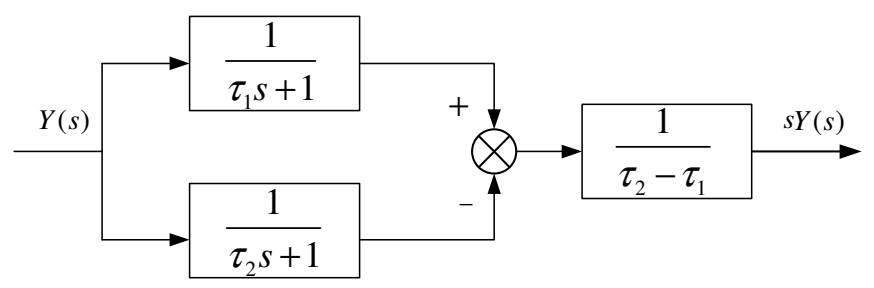

Fig. 3 Schematic structure of the enhanced differentiator

Similar to the above analysis, the actual derivative $\dot{\hat{y}}$ of the enhanced differentiator with the noise $\eta$ can be described as follows, 


$$
\begin{aligned}
s \hat{Y}(s) & =\frac{s}{\tau_{1} \tau_{2} s^{2}+\left(\tau_{1}+\tau_{2}\right) s+1}(Y(s)+\eta) \\
& =\frac{s}{\tau_{1} \tau_{2} s^{2}+\left(\tau_{1}+\tau_{2}\right) s+1} y+\frac{s}{\tau_{1} \tau_{2} s^{2}+\left(\tau_{1}+\tau_{2}\right) s+1} \eta \\
& =s Y(s)+\frac{s}{\tau_{1} \tau_{2} s^{2}+\left(\tau_{1}+\tau_{2}\right) s+1} \eta
\end{aligned}
$$

The output of the proposed differentiator can be further given as $s \hat{Y}(s)=s Y(s)+\frac{\frac{1}{\tau^{2}} s}{\left(s+\frac{1}{\tau}\right)^{2}} \eta$, when time constants $\tau_{1}$ and $\tau_{2}$ are close to system time delay constant $\tau$. Following the terminal value theorem, the last polynomial related to noise $\eta$ can be further analyzed:

$$
\dot{\hat{y}}(\infty)=\lim _{s \rightarrow 0} \dot{y}(\infty)+\lim _{s \rightarrow 0}\left(\frac{\frac{1}{\tau^{2}} s}{\left(s+\frac{1}{\tau}\right)^{2}} \eta\right) \approx \lim _{s \rightarrow 0} \dot{y}(\infty)
$$

TSe the noise $\eta$ can be minimized by the proposed differential method in Eq. (15). Similar to the first mode vibration output of the all-clamped piezoelectric plate, a frequency $f=51.9 \mathrm{~Hz}$ sinusoidal signal with an amplitude \pm 1 is mixed with $10 \mathrm{kHz}$ noise with an amplitude of \pm 0.01 , in order to verify the effectiveness and superiority of the proposed enhanced differentiator. This mixed signal is processed by the traditional and the proposed differentiators, where the comparison results and the $2 \pi f$ times of the original signal are given in Fig. 4. It indicates that the noise is obviously amplified by the traditional differentiator, while the proposed differentiator can effectively extract the required differential signal with excellent noise rejection performance. 


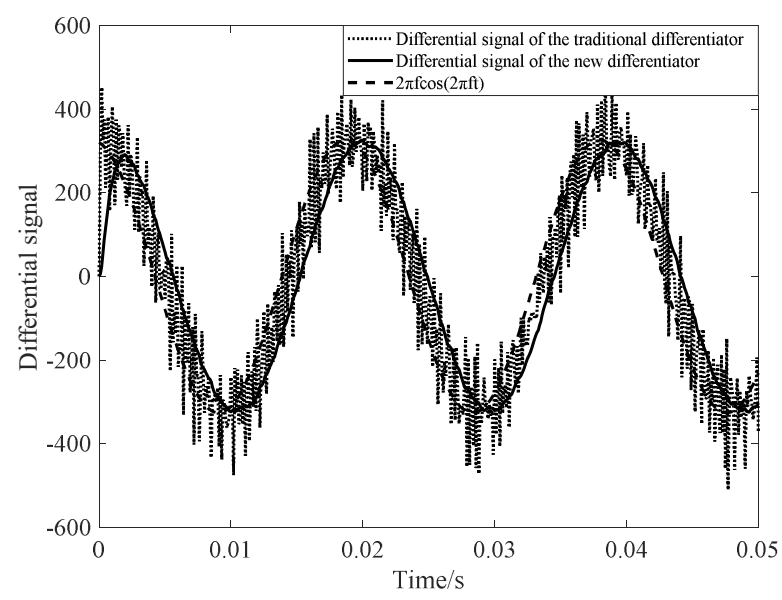

Fig. 4 Comparison of the traditional and proposed differentiators

\subsection{ADRC for All-Clamped Piezoelectric Structure}

State space equation of the single mode vibration Eq. (6) can be put into Eq. (16):

$$
\left\{\begin{array}{l}
\dot{x}_{1}=x_{2} \\
\dot{x}_{2}=-c_{2} x_{1}-c_{1} x_{2}+w+c_{0} u \\
y=x_{1}
\end{array}\right.
$$

where parameter $w$ represents the external disturbances including the external excitation. Parameters $c_{1}$ and $c_{2}$ denote system coefficients. Symbol $c_{0}$ is a nominal system parameter. System state variables $x_{1}$ and $x_{2}$ are the model displacement and velocity, respectively. The system internal and external disturbances can be defined by Eq. (17):

$$
f=-c_{2} x_{1}-c_{1} x_{2}+w-\left(\hat{c}_{0}-c_{0}\right) u,
$$

where the polynomial $-c_{2} x_{1}-c_{1} x_{2}-\left(\hat{c}_{0}-c_{0}\right) u$ is considered as the internal disturbances. The total disturbances, including external excitations, model error, uncertainty dynamics and vibration of system parameters are expressed by $f$. The symbol $\hat{c}_{0}$ represents the estimated value of $c_{0}$. The second state variable of the vibration system can be simplified as: 


$$
\dot{x}_{2}=f+\hat{c}_{0} u \text {. }
$$

The basic idea of ADRC based vibration control is to estimate the total disturbances $f$ by ESO and to actively compensate for the disturbances with feed-forward control part. An extended state $x_{3}=f$ is introduced to express the total disturbances with $h=\dot{f}$. Assuming that $f$ is globally differentiable, the system state space model can be rewritten as an extended state equation.

$$
\left\{\begin{array}{l}
\dot{x}_{1}=x_{2} \\
\dot{x}_{2}=x_{3}+\hat{c}_{0} u \\
\dot{x}_{3}=h \\
y=x_{1}
\end{array} .\right.
$$

Similar to refs. [48-50], the ESO corresponding to Eq. (19) can be designed as:

$$
\left\{\begin{array}{l}
e_{1}=z_{1}-y \\
\dot{z}_{1}=z_{2}-3 \beta e_{1} \\
\dot{z}_{2}=z_{3}-3 \beta^{2} e_{1}+\hat{c}_{0} u \\
\dot{z}_{3}=-\beta^{3} e_{1}
\end{array},\right.
$$

where $z_{1}$ and $z_{2}$ are observer state estimating system state variables $x_{1}$ and $x_{2}$, respectively. Parameter $e_{1}$ is the estimated error between the actual output and observer output of the ESO. The variable $z_{3}$ is the estimation of the total disturbances $f$. In traditional LADRC based vibration control system, the parameter $\beta$, which is defined as the observer bandwidth of ESO, should be reasonably selected by making a tradeoff between vibration control performance and noise tolerance. Fortunately, the proposed time delay compensation based LADRC (TDCLADRC) vibration control with the novel differentiator can effectively attenuate the effect of noise, so the design of the vibration controller is simple and easy to implement compared against the conventional ADRC-based vibration control methods. By 
canceling the estimated error of $z_{3}$ and $f$, the composite control value $u$ can be given by Eq. (21) with well selected observer bandwidth $\beta$ :

$$
u=u_{0}-\frac{z_{3}}{\hat{c}_{0}}
$$

The second equation of Eq. (19) can be further rewritten in Eq. (22) by substituting Eq. (21) into Eq. (19):

$$
\dot{x}_{2}=f\left(x_{1}, x_{2}, w\right)+\hat{c}_{0}\left(u_{0}-\frac{z_{3}}{\hat{c}_{0}}\right) \approx \hat{c}_{0} u_{0} .
$$

The piezoelectric plate structural modal vibration can be further converted into an integral series system, since the total disturbances $f$ can be observed and compensated through the estimated value $z_{3}$ from ESO. So the traditional PD controller, expressed in Eq. (23), is employed for the vibration suppression with the estimated values of modal displacement $z_{1}$ and modal velocity $z_{2}$.

$$
\left\{\begin{array}{l}
u=u_{0}-\frac{z_{3}}{\hat{c}_{0}} \\
u_{0}=k_{p}\left(0-z_{1}\right)-k_{d}\left(0-z_{2}\right)=-k_{p} z_{1}-k_{d} z_{2}
\end{array},\right.
$$

where $k_{p}$ and $k_{d}$ are the feedback gains. The LADRC-based vibration control law, expressed by the first equation of Eq. (23), can be obtained by substituting the second equation of Eq. (23) into Eq. (22). Additionally, the stability of the entire structural vibration system can be guaranteed by proper feedback gains $\left(k_{p}\right.$ and $\left.k_{d}\right)$ of PD controller and bandwidth $\beta$ of ESO. The system time delay is not considered in the control law (23), but the system time delay caused by mismatched sensor/actuator pairs, computational delay, and the sampling time of acquisition card, is inevitable in 
practical structural vibration control systems. Unfortunately, this problem may lead to energy loss due to the untimely control input value, or even destroy the overall system stability. So the vibration suppression method based on LADRC with the proposed enhanced smith predictor algorithm, i.e., time delay compensation for LADRC (TDCLADRC), can solve the problem to enhance the vibration suppression performance according the vibration acquisition characteristics of the piezoelectric plate structures. This proposed TDCLADRC vibration control method can be obtained in Eq. (24) and shown in Fig. 5.

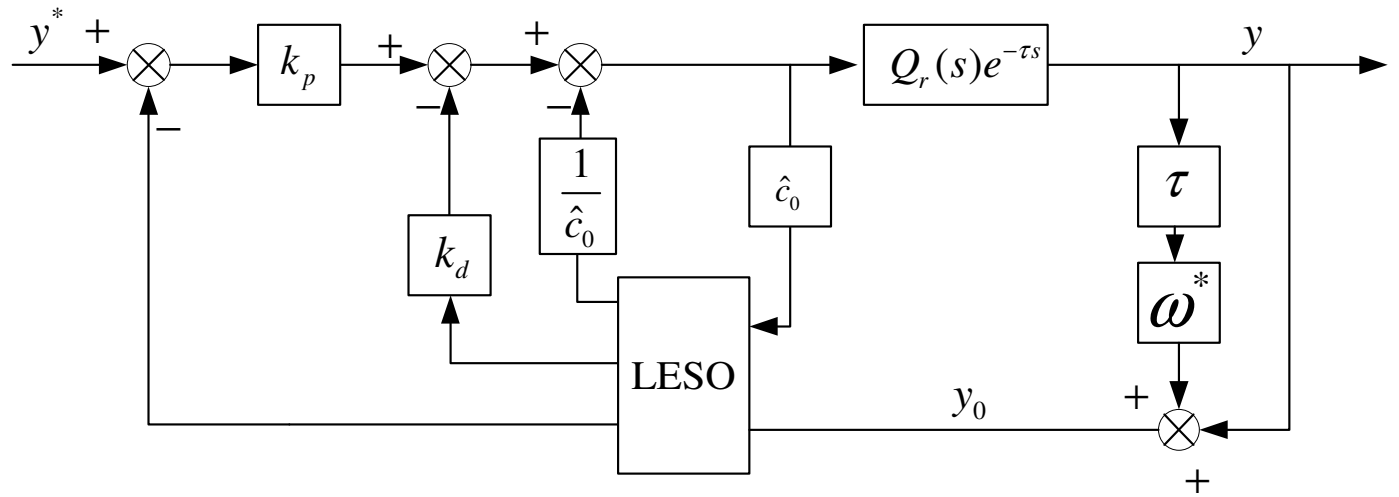

Fig. 5 Schematic diagram of the proposed TDCLADRC

$$
\left\{\begin{array}{l}
e_{2}=z_{1}-y_{0} \\
\dot{z}_{1}=z_{2}-3 \beta e_{2} \\
\dot{z}_{2}=z_{3}-3 \beta^{2} e_{2}+\hat{c}_{0} u \\
\dot{z}_{3}=-\beta^{3} e_{2} \\
y_{0}=y+\tau \omega^{*} y \\
u=-k_{p} z_{1}-k_{d} z_{2}-\frac{z_{3}}{\hat{c}_{0}}
\end{array},\right.
$$

where $e_{2}$ is the estimated error between the observer value and auxiliary output $y_{0}$ obtained by the actual output $y$ and its differential.

\section{Experimental Verifications}

\subsection{Experimental Set-up}

The all-clamped plate structure (aluminum alloy LY12CZ) with a dimension of 
$500 \mathrm{~mm} \times 500 \mathrm{~mm} \times 1 \mathrm{~mm}$, is excited by a vibrator $(\mathrm{HEV}-20)$ located at the right of the plated, as shown in Fig. 6. The clamped condition is achieved by putting four side ends of the thin plate between the steel bars bonded together tightly with several bolts. The first-order natural frequency of the piezoelectric thin plate is $51.9 \mathrm{~Hz}$, which is obtained by the hammering method with a force hammer (LC-02A). The piezoelectric bimorph with a size of $40 \mathrm{~mm} \times 10 \mathrm{~mm} \times 0.8 \mathrm{~mm}$ is located at the surface of the middle of the plate structure, where the maximum strain of the first mode is induced and first several modes can be measured. An accelerometer (IEPE-CA-YD-160), close to piezoelectric bimorph and with a constant current source regulator (IEPE-YE3821), is employed to measure the vibration response. The vibration signal from the accelerometer is sampled with the I/O module of NI-PCIe6343, and then transmitted to the analog input module in real-time environment under Maltab/Simulink environment. The control value is calculated by the corresponding algorithms (including the proposed TDCLADRC in this paper). Then this control action is amplified to the peak-to-peak voltage of $\pm 100 v$ with a power amplifier (HVP-300D) which drives the piezoelectric bimorph to suppress the vibration.

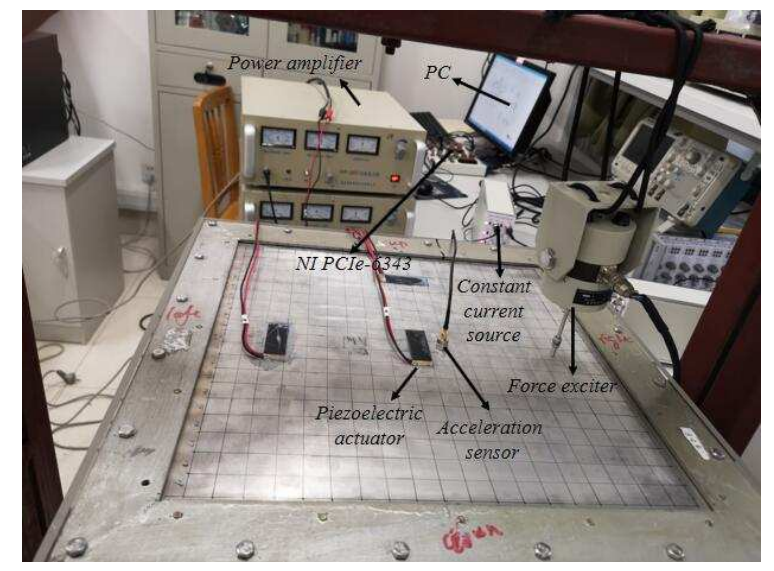

Fig. 6 Configuration of experimental set-up

\subsection{System Identification Experiment}



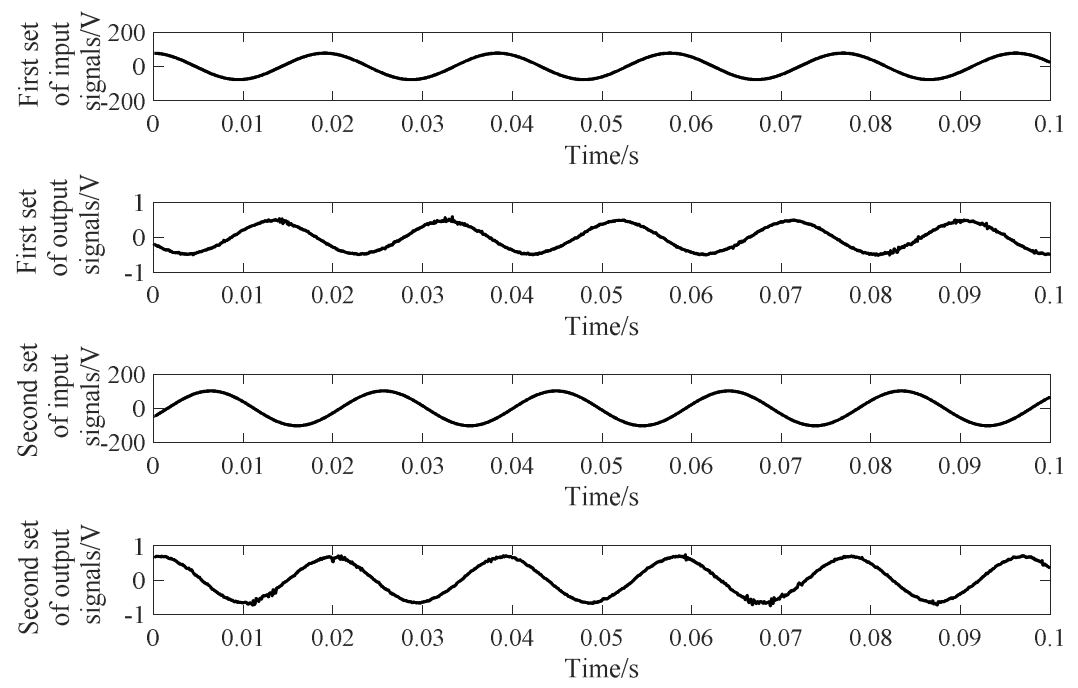

Fig. 7 Two sets of input and output data from acquisition system

These two sets of data in Fig. 7 are acquired by the I/O module of NI-PCIe6343 with the sampling frequency of $10 \mathrm{kHz}$. In order to obtain an accurate structural vibration model, one set of data is used for system model identification, while the other is for model verification and correction. The phase lag of the vibration model caused by the no-collocated sensor/actuator pair, computational delay, and sampling time, may decrease the vibration suppression performance or even lead to system instability. In order to verify the excellent performance of the proposed TDCLADRC approach, the time delay coefficient $\tau$ should be identified before system modeling. So a persistent excitation at the first resonant frequency, i.e., $51.9 \mathrm{~Hz}$, is carried out for the phase hysteresis identification. The force vibrator with the power amplifier is also used to excite persistent vibration of the all-clamped thin piezoelectric plate structure. The experimental data from the power amplifier of force vibrator and the accelerometer acquired by the acquisition card, can be recorded for a period of time when the excited modal vibration is stable and remains at a fixed amplitude. The Lissajous curves, shown in Fig. 8, can be plotted by using these two sets of data as Xand Y-coordinates, respectively. It follows from Fig. 8 that the phase hysteresis phenomenon exists in the structural vibration system, since the hysteresis loop resembles an ellipse. In order to estimate the time delay coefficient of the whole control system, the Lissajous curves for the vibration mode is fitted by Eq. (25):

$$
\begin{aligned}
& x=76.4 \sin (2 \pi H t) \\
& y=0.48 \sin (2 \pi H t+\varphi)
\end{aligned}
$$




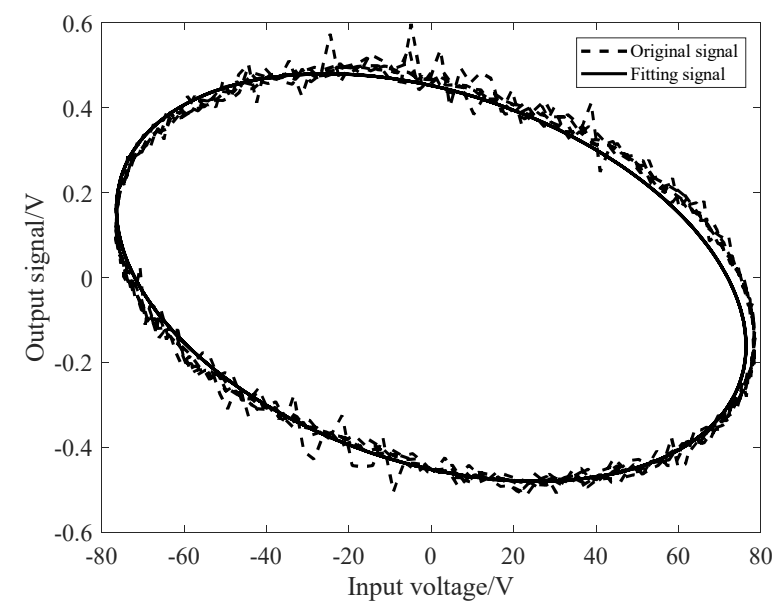

Fig. 8 Lissajous curves of the vibration system between the signals measured from power amplifier and accelerometer

where the natural frequency is $H=51.9 \mathrm{~Hz}$ and the phase error is $\varphi=4.37 \mathrm{rad}$, since the curve-fitting Lissajous figure can be obtained as the solid line in Fig. 8, by introducing Eq. (25) as the $\mathrm{X}$ - and the $\mathrm{Y}$ - coordinates. In addition, the time delay of the whole vibration control system can be obtained by Eq. (26) with the above phase lag identification results.

$$
\tau=\frac{1000 \varphi}{2 \pi H}=13.41 \mathrm{~ms}
$$

Considering that the identified second-order vibration system itself has two first-order inertial links, the system time delay constant should be reserved at a certain margin. Therefore, a second-order system transfer function, expressed in Eq. (27), is identified with the highest fitting degree in the range of $0 \leq \tau \leq 0.01341$.

$$
Q_{r}(s)=\frac{y(s)}{u(s)}=\frac{74.57}{s^{2}+12.72 s+95230} e^{-0.005 s} .
$$




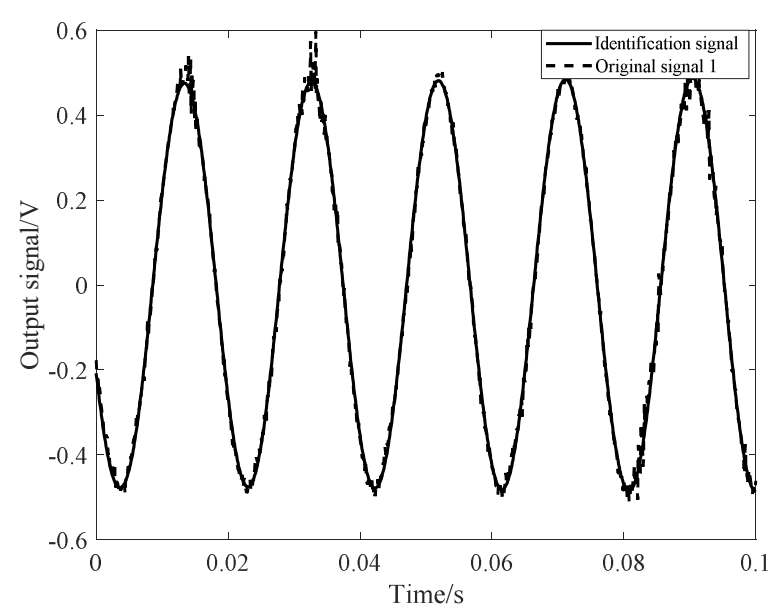

Fig. 9 Data fitting curves between the first set data and output of Eq. (27)

The data fitting curves between the original acquisition data and Eq. (27) are shown in Figs. 9. It follows from Fig. 9 that the experimental data and transfer function (27) fit very well. In order to further verify the modeling accuracy, the second set input data in Fig. 7 is given to the transfer function (27). The input/output fitting-curves of the Eq. (27) and the second set of the original data is shown in Fig. 10. It follows from Fig. 10 that the fitting degree of the two curves is also relatively high, which again verifies the effectiveness of the open-loop transfer function in Eq. (27).

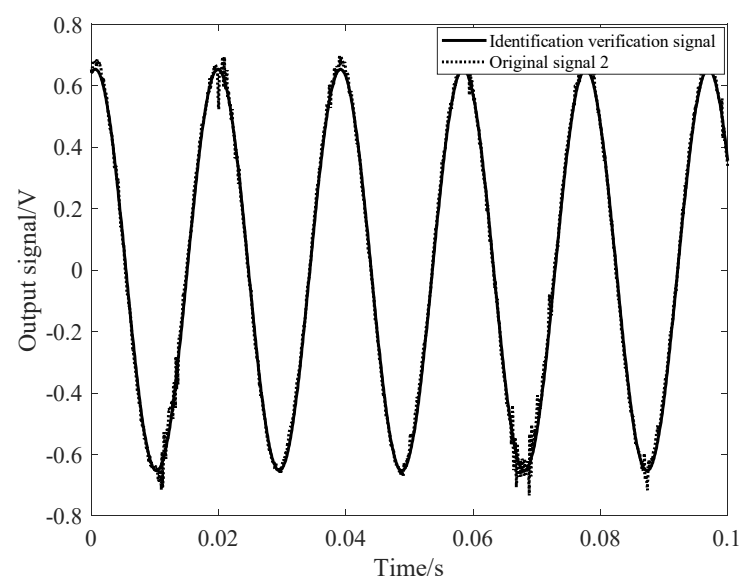

Fig. 10 Data fitting curves between the second set data and output of Eq. (27)

\subsection{Vibration Control Experiment}

In order to verify the effectiveness and superiority of the proposed TDCLADRC based vibration control method, the comparative experiments against the traditional LADRC and predictor based LADRC (PLADRC) are conducted for the all-clamped thin piezoelectric plate, which is excited by the first modal natural frequency. Similar 
as ref. [48], the spectrum of the normalized acceleration signal is defined by Eq. (28) for easy comparison:

$$
\text { The decibel value }=20 \log _{10}\left(\xi\left(y / y_{R}\right)\right) \text {, }
$$

where the function $\xi(\cdot)$ represents the Fourier Transformation. The reference vibration output signal for normalization is expressed by $y_{R}$. The value of $y_{R}$ is set to be $1 \mathrm{~g}$ as the standard value, i.e., $0 \mathrm{~dB}$ is equivalent to $1 \mathrm{~g}$ and $-20 \mathrm{~dB}$ is equivalent to $0.1 \mathrm{~g}$.

Firstly, results for the traditional LADRC is presented, which-were the time and frequency responses of the traditional LADRC and free-controller for the plate structure are shown in Figs. 11 and 12, respectively. It can be seen that the all-clamped piezoelectric plate is excited by the harmonic signal. Additionally, the response curves in Fig. 11 show that the acceleration sensor with the traditional LADRC is less than one-fourth of the original value without controller. Since the ESO estimates the internal and external disturbances of the piezoelectric plate, and attenuates the effects of the disturbances through feed-forward channel, the LADRC can effectively suppress the structural vibration.
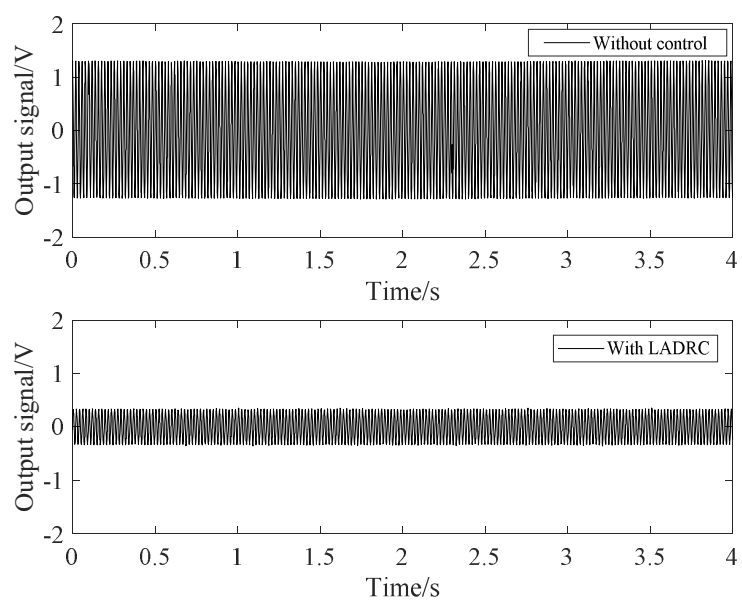

Fig. 11 Suppression performance in time domain under LADRC and without controller. 


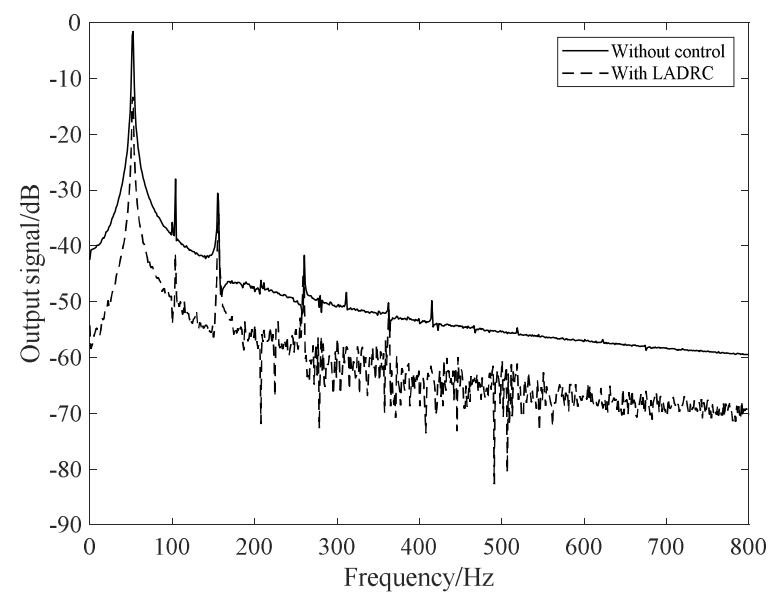

Fig. 12 Suppression performance in frequency domain under LADRC and without controller.

In addition, the three control methods, i.e., LADRC, PLADRC, and the proposed TDCLADRC, are also applied for the all-clamped piezoelectric plate structure excited by a force vibrator in Fig. 13. The following conditions are chosen for the three ADRC-based vibration controllers to make a fair comparison. Firstly, the excitation condition has the same frequency and almost the same magnitude voltage; secondly, the piezoelectric actuators, the accelerometer, and force vibrator are always the same as the collocated way; thirdly, all the active vibration control algorithms are simulated by the real-time module in Matlab2018, which is implemented by a NI-PCIe6343 board, as shown in Fig. 6. 


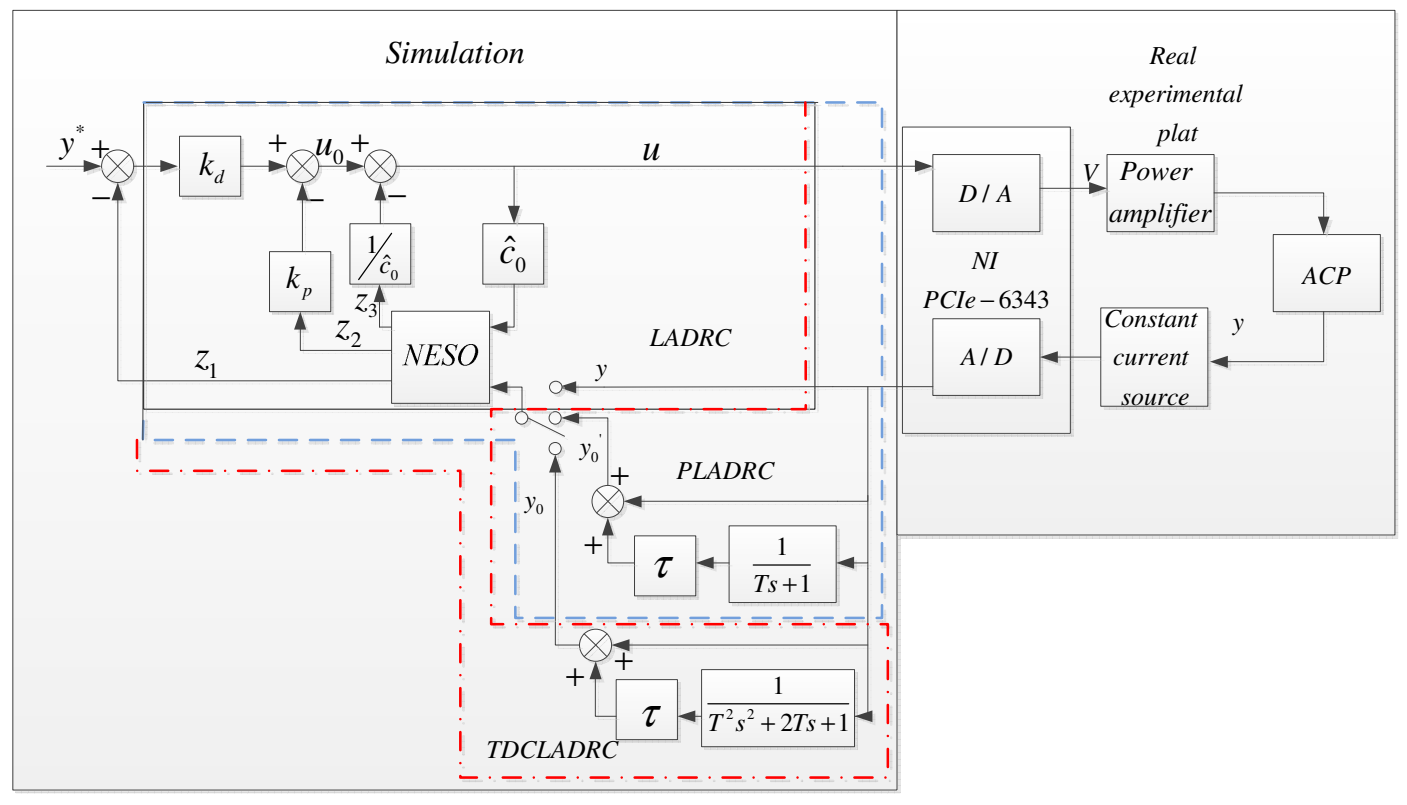

Fig. 13 Diagram of the LADRC, PLADRC and TDCLADRC controllers for an all-clamped plate structural vibration

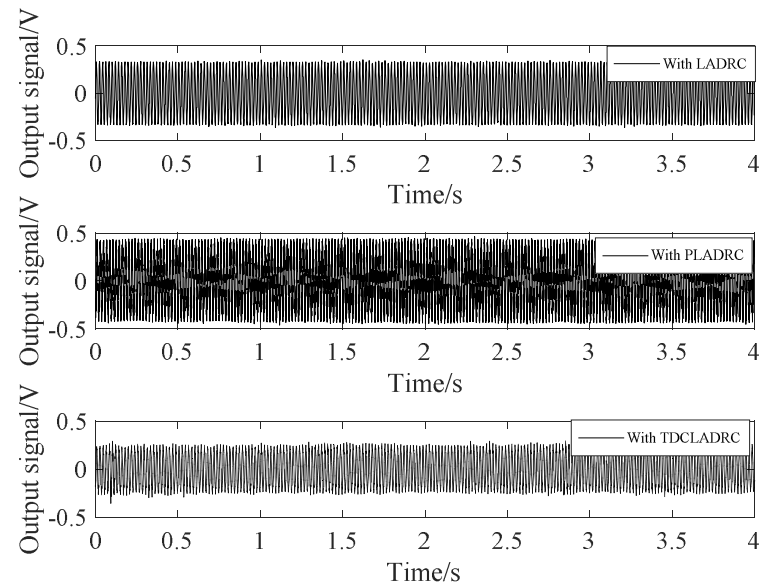

(a) Globe response curves under the three control methods

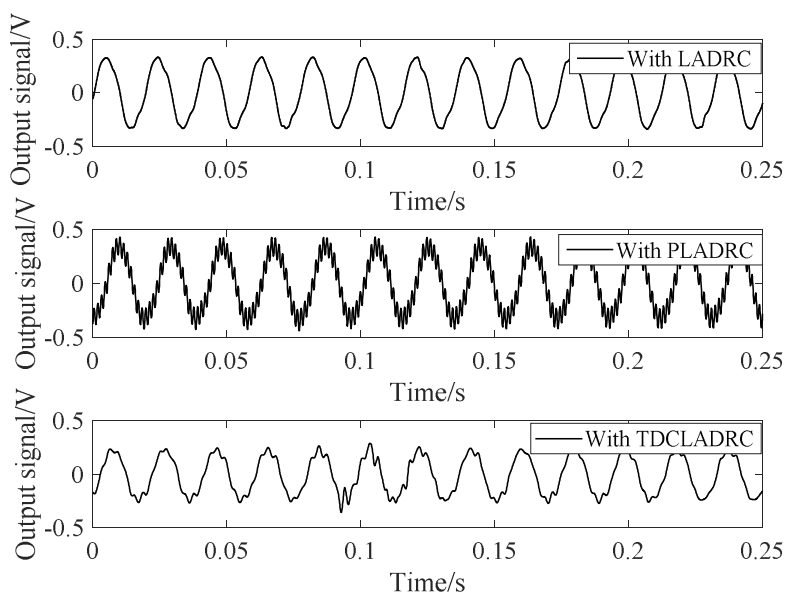

(b) Local response curves under the three control methods 
Fig. 14Vibration suppression performance comparisons in time domain under traditional LADRC, PLADRC and the proposed TDCLADRC.

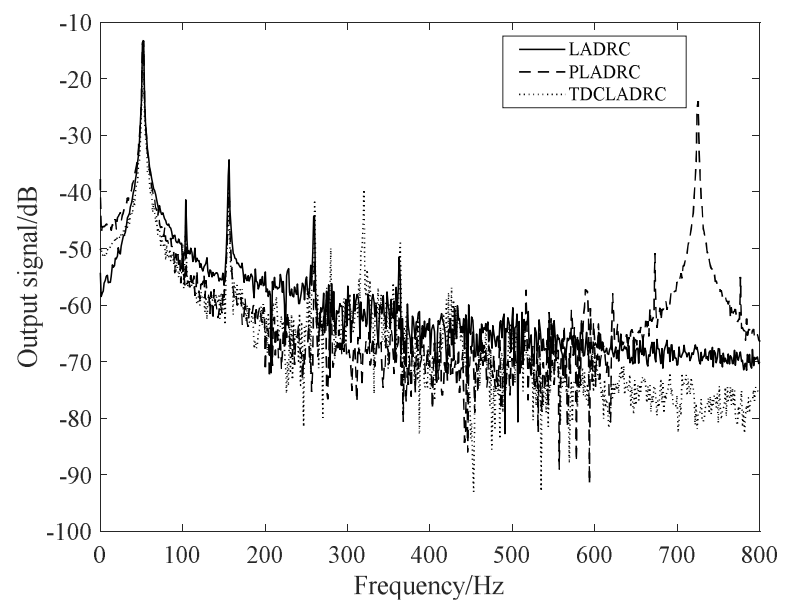

Fig. 15 Vibration suppression performance comparisons in frequency domain under traditional LADRC, PLADRC and the proposed TDCLADRC.

In order to achieve excellent vibration suppression performance, the control parameters including $\hat{c}_{0}$ and $\beta$ should be reasonably selected by considering the output limitation of the I/O module of NI-PCIe6343. The control parameters of ADRC-based vibration controller, i.e., $k_{p}=12, k_{d}=1.25, \quad \beta=12, \hat{c}_{0}=40$, are chosen to make a tradeoff between vibration suppression performance and system hardware limitation.

The time and frequency domain responses of the three control methods are shown in Figs. 14 and 15. Additionally, it follows from Figs. 14 and 15 that the vibration suppression level described by the spectrum of acceleration signal is reduced almost in the whole frequency range. From the experimental results shown in Fig. 14, it is also illustrated that the whole vibration suppression performance by the proposed TDCLADRC-based vibration control method outperforms other approaches. It follows from Fig. 16 the control voltages of the piezoelectric bimorph under the 
three ADRC-based control methods are with almost the same amplitude.

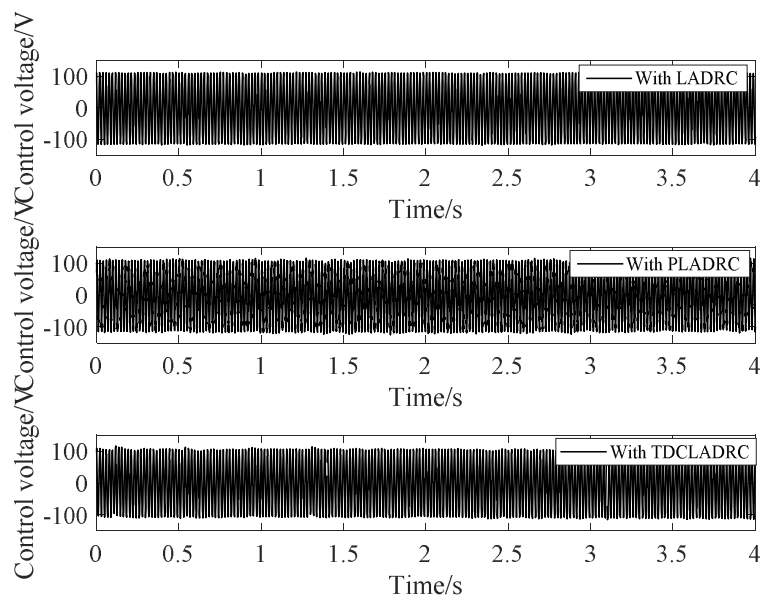

(a) Piezoelectric bimorph driven voltages

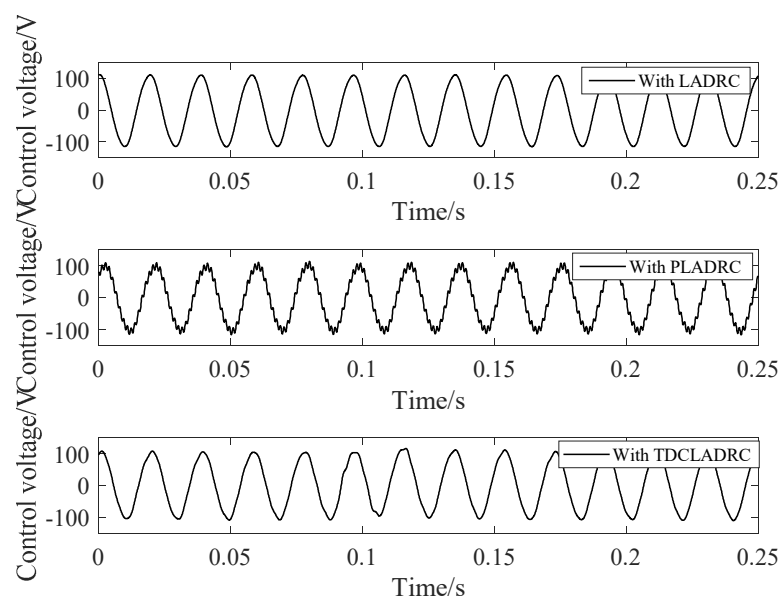

(b) Local curves of the driven voltages

Fig. 16 Experimental control voltage curves under traditional LADRC, PLADRC and the proposed TDCLADRC.

Along with the control voltages in Fig. 16-and the frequency domain responses in Fig. 16, it can be seen that the first mode of the all-clamped piezoelectric plate can be reduced by $11.7 \mathrm{~dB}, 11.57 \mathrm{~dB}$ and $13.43 \mathrm{~dB}$ under the traditional LADRC, PLADRC, and the proposed TDCLADRC methods, respectively. The vibration suppression performances of the classical LADRC, PLADRC and the proposed TDCLADRC for 
the all-clamped piezoelectric plate are summarized in Table 1. The decibel values based on Eq. (28) of the first five peaks without and with control are listed.

Table1 Vibration suppression performances of the LADRC, PLADRC and the proposed TDCLADRC for piezoelectric plate

\begin{tabular}{|l|ccccc|c|}
\hline Frequency $(\mathrm{Hz})$ & 51.9 & 103.8 & 156.3 & 319.9 & 725.3 & Control voltage(V) \\
No control $(\mathrm{dB})$ & -1.59 & -28.04 & -32.08 & -51.29 & -58.66 & \\
LADRC $(\mathrm{dB})$ & -13.29 & -41.38 & -34.31 & -60.73 & -68.59 & 110.6 (peak value) \\
PLADRC $(\mathrm{dB})$ & -13.16 & -47.49 & -38.39 & -68.55 & -23.49 & 109.3 (peak value) \\
TDCLADRC(dB) & -15.02 & -45.71 & -37.30 & -39.57 & -78.94 & 107.9 (peak value) \\
\hline
\end{tabular}

From Fig. 16 (b), it is easily obtained that the control values based on the PLADRC and TDCLADRC are a little quicker than those under the traditional LADRC-based vibration method, that which is because the time delay is considered in the control design. Unfortunately, a contradictory experimental result is obtained to indicate that the vibration suppression performance under the traditional LADRC is better than those under the PLADRC based on the traditional differentiator from Fig. 14 (a). Further analysis of Fig. 16 (b) and Fig. 14(b) shows that the control signal based on the PLADRC with the traditional differentiator has been polluted by noise due to the inevitable accelerometer noise.

It is also shown in Fig. 15 that there exists a high amplitude fluctuation at the 725.3Hz with PLADRC. The PLADRC with a traditional differentiator amplifies the frequency acceleration signal, and as a result its performance is slightly worse than the traditional LADRC. In addition, although the traditional LADRC approach has a certain time delay margin and noise tolerance under suitable control parameters, its vibration suppression performance is very limited in many conditions. In addition, the proposed TDCLADRC method in Eq. (24) with the novel differentiator Eq. (14) can achieve excellent vibration suppression performance for the all-clamped piezoelectric thin plate, which is mainly due to its strong disturbance rejection and differential 
estimation abilities. Thus, the proposed TDCLADRC with the novel differentiator method is an effective and superior method to overcome the structural vibration problems with internal and external disturbances, time delay and complex boundary conditions.

\section{Conclusions}

Although the traditional LADRC based vibration control allows for certain time delay and has an excellent anti-disturbance ability for complex structural vibration control system, its vibration suppression performance will be degraded in the presence of internal and external disturbances, inevitable phase lag and sensor noise, simultaneously. To achieve satisfying vibration suppression performance, a time delay compensated based LADRC with a novel differentiator is introduced, analyzed, and applied to an all-clamped piezoelectric thin plate. Several strategies have been introduced into the ADRC-based vibration control methods to enhance its vibration suppression performance. First, an auxiliary variable method with Lissajou algorithm is introduced to identify and correct the modal vibration of the plate structure. Second, a novel differentiator technology is drawn to address the problems of system time delay and sensor noise. Compared with the traditional LADRC and the predictor based ADRC methods, comparative experimental results on an all-clamped piezoelectric plate illustrate that the vibration acceleration amplitudes of the three modes are reduced by $11.7 \mathrm{~dB}, 11.57 \mathrm{~dB}$ and $13.43 \mathrm{~dB}$ at the first order resonance frequency point, respectively. The experimental results show that the performance of the traditional LADRC based vibration controller is better than the PLADRC with traditional differentiator, since the PLADRC amplifies the sensor noise in calculating the differential computation. Therefore, in order to enhance the control performance of the system with time delay, the structure and the parameters of the time delay 
compensation method should be reasonably chosen to embed the ADRC-based vibration controller in actual vibration suppression system.

\section{Acknowledgements}

This work was supported in part by the National Natural Science Foundation of China (Grant nos. 61773335, 61903322, 51975266), Natural Science Foundation of Jiangsu Province (Grant no. BK20171289), State Key Laboratory of Mechanics and Control of Mechanical Structures (Grant No. MCMS-E-0520G01), Six Talent Peaks Foundation of Jiangsu Provincial (Grant no. KTHY2018038), the Natural Science Foundation of Yangzhou City for Outstanding Young Scholars (Grant no. YZ2017099).

\section{Conflict of interest}

None declared.

\section{References}

[1] Kumar Y. The Rayleigh-Ritz method for linear dynamic, static and buckling behavior of beams, shells and plates: A literature review. Journal of Vibration and Control, 24(7): 1205-1227, 2018.

[2] Thai HT, Kim SE. A review of theories for modeling and analysis of functionally graded plates and shells. Composite Structures, 128: 70-86, 2015.

[3] Zhang SQ, Zhao GZ, Rao MN, et al. A review on modeling techniques of piezoelectric integrated plates and shells. Journal of Intelligent Material and Structures, 30(8): 1133-1147, 2019.

[4] Feliu-Talegon D, San-Millan A, Feliu-Batlle V. Fractional-order integral resonant control of collocated smart structures. Control Engineering Practice 56: 210-223, 2016.

[5] Rosenzweig P, Kater A, Meurer T. Model predictor control of piezo-actuator structures using reduced order models. Control Engineering Practice, 80: 83-93, 2018.

[6] Wu YF, Deng MC. Operator-based robust nonlinear optimal vibration control for an L-shaped arm driven by linear pulse motor. International Journal of Control Automation and Systems, 15(5): 2026-2033, 2017.

[7] Pu YX, Zhou HL, Meng Z. Multi-channel adaptive active vibration control of piezoelectric smart 
plate with nonlinear secondary patch modelling using PZT patches. Mechanical Systems and Signal Processing, 120: 167-179, 2019.

[8] Sun N, Liang DK, Wu YM, et al. Adaptive control for pneumatic artificial muscle systems with parametric uncertainties and unidirectional input constraints. IEEE Transactions on Industrial Informatics, 16(2): 969-979, 2020.

[9] Goncaives JF, De Leon DM, Perondi EA. Topology optimization of embedded piezoelectric actuators considering control spillover effects. Journal of Sound and Vibration, 388: 20-41, 2017.

[10] Wang S, Chen ZB, Liu XX, et al. Feedforward feeback linearization linear quadratic Gaussian with loop transfer recovery control of piezoelectric actuator in active vibration solution system. Journal of Vibration and Acoustics-Transaction of The ASME, 140(4): 041009, 2018.

[11] Al Janaideh M, Rakotondrabe M, Al-Darabsah I, et al. Internal model-based feedback control design for inversion-free feedforward rate-dependent hysteresis compensation of piezoelectric cantilever actuator. Control Engineering Practice, 72: 29-41, 2018.

[12]Zhang YM, Yan P, Zhang Z. Robust adaptive backstepping control for piezoelectric nano-manipulating systems. Mechanical Systems and Signal Processing, 83: 130-148, 2017.

[13] Simonovic AM, Jovanovic MM, Lukic NS, et al. Experimental studies on active vibration control of smart plate using a modified PID controller with optimal orientation of piezoelectric actuator. Journal of Vibration and Control, 22(11): 2619-2631, 2016.

[14]Zhang SQ, Schmidt R, Qin XS. Active vibration control of piezoelectric bonded smart structures using PID algorithm. Chinese Journal of Aeronautics, 28(1): 305-313, 2015.

[15] Tehrani MG, Elliott RNR, Mottershead JE. Partial pole placement in structures by the method of receptances: Theory and experiments. Journal of Sound and Vibration, 329(24): 5017-5035, 2010.

[16]Li FM, Yao G, Zhang YM. Active control of nonlinear forced vibration in a flexible beam using piezoelectric material. Mechanics of Advanced Materials and Structures, 23(3): 311-317, 2016.

[17] Chuaqui TRC, Roque CMC, Riheiro P. Active vibration control of piezoelectric smart beams with radial basis function generated finite difference collocation method. Journal of Intelligent Material Systems and Structures, 29(13): 2728-2743, 2018.

[18]Zhang XW, Wang CX, Liu J, et al. Robust active control based milling chatter suppression with perturbation model via piezoelectric stack actuators. Mechanical Systems and Signal Processing, 120: 808-835, 2019. 
[19] Jiaqiang EQ, Qian C, Liu HL, et al. Design of the H-infinity robust control for the piezoelectric actuator based on chaos optimization algorithm. Aerospace Science and Technology, 47: 238-246, 2015.

[20]Lin CY, Huang YH, Chen WT. Multimodal suppression of vibration in smart flexible beam using piezoelectric electrode-based switch control. Mechatronics, 53: 152-167, 2018.

[21] Yue HH, Lu YF, Deng ZQ, et al. Experiments on vibration control of a piezoelectric laminated paraboloidal shell. Mechanical Systems and Signal Processing, 82: 279-295, 2017.

[22] Lin CY, Huang YH. Enhancing vibration suppression in a periodically excited flexible beam by using a repetitive model predictive control strategy. Journal of Vibration and Control, 22(16): 3518-3531, 2016.

[23] Rosenzweig P, Kater A, Meurer T. Modal predictive control of piezo-actuated structures using reduced order models. Control Engineering Practice, 80: 83-93, 2019.

[24] Kim B, Washington GN, Yoon HS. Active vibration suppression of a 1D piezoelectric bimorph structures using modal predictive sliding mode control. Smart Structures and Systems, 11(6): 623-635, 2013.

[25] Qiu ZC, Zhang SM. RBF neural network-based sliding vibration control of a flexible cantilever plate using laser displacement measurement. Proceeding of the Institution of Mechanical Engineers Part G- Journal of Aerospace Engineering, 231(8): 1492-1508, 2017.

[26] Sun N, Wu YM, Chen H, et al. Antiswing cargo transportation of underactuated tower crane systems by a nonlinear controller embedded with an integral term. IEEE Transactions on Automation Science and Engineering, 16(3): 1387-1398, 2019.

[27] Cao XB, Yue CF, Liu M. Flexible satellite attitude maneuver via constrained torque distribution and active vibration suppression. Aerospace Science and Technology, 67: 387-397, 2017.

[28] Yang J, Zheng WX. Offset-free nonlinear MPC for mismatched disturbance attenuation with application to a static var compensator. IEEE Transactions on Circuits and Systems II-Express Briefs, 61(1): 49-53, 2014.

[29] Han JQ. From PID to active disturbance rejection control. IEEE Transactions on Industrial Electronics, 56(3): 900-906, 2009.

[30] Ohnishi K. A new servo method in mechatronics. Transactions of Japanese Society Electronics Engineering, 107(D): 83-86, 1987. 
[31] Li SQ, Li J, Qiu JH, et al. Multi-modal vibration control using amended disturbance observer compensation. IET Control Theory and Applications, 6(1):72-83, 2012.

[32] Liu Y, Fu Y, He W, et al. Modeling and observer-based vibration control of a flexible spacecraft with external disturbances. IEEE Transactions on Industrial Electronics, 66(11):8648-8658, 2019.

[33] Tang T, Niu SX, Chen XQ, et al. Disturbance observer-based control of tip-tilt mirror for mitigating telescope vibrations. IEEE Transactions on Instrumentation and Measurement, 68(8): 2785-2791, 2019.

[34] He W, Ge SS. Vibration control of a nonuniform wind turbine tower via disturbance observer. IEEE-ASME Transactions on Mechatronics, 20(1): 237-244, 2015.

[35] Pan CK, Lewis FL, Ge SS, et al. Singular perturbation control for vibration rejection in HDDs using the PZT active suspension as fast subsystem observer. IEEE Transactions on Industrial Electronics, 54(3): 1375-1386, 2007.

[36] Gilbergs H, Fang H, Frenner K, et al. Adaptive state observer and PD control for dynamic perturbations in optical systems. Optics Express, 23(4): 4002-4011, 2015.

[37] She JH, Fang MX, Ohyama Y, et al. Improving disturbance-rejection performance based on an equivalent-input-disturbance approach. IEEE Transactions on Industrial Electronics, 55(1): $380-389,2008$

[38] Miyamoto K, She JH, Imani J, et al. Equivalent-input-disturbance approach to active structural control for seismically excited building. Engineering Structures, 125(10): 392-399, 2016.

[39]Zhu Q, Yue JZ, Liu WQ, et al. Active vibration control for piezoelectricity cantilever beam: an adaptive feedforward control method. Smart Materials and Structures, 26(4): 047003, 2017.

[40] Ribic AI, Despotovic ZV. High-performance feedback control of electromagnetic vibratory feeder. IEEE Transactions on Industrial Electronics, 57(9): 3087-3094, 2010.

[41] Morales R, Feliu V, Jaramillo V. Position control of very lightweight single-link flexible arms with large payload variations by using disturbance observers. Robotics and Autonomous Systems, 60(4): $532-547,2012$.

[42]Zhang SQ, Li HN, Schmidt R, et al. Disturbance rejection control for vibration suppression of piezoelectric laminated thin walled structures. Journal of Sound and Vibration, 333(5): 1209-1223, 2014.

[43]Zhong QC, Rees D. Control of uncertain LTI systems based on an uncertainty and disturbance 
estimator. Journal of Dynamic Systems Measurement and Control-Transactions of the ASME, 126(4): 905-910, 2004.

[44] Kodhanda A, All N, Sucheendran MM, et al. Robust control of nonlinear resonance in a clamped rectangular plate. Journal of vibration and Control, 24(18): 4176-4194, 2018.

[45] Dai JG, Ren BB. UDE-based robust boundary control for an unstable parabolic PDE with unknown input disturbance. Automatica, 93: 363-368, 2018.

[46]Zhang H, Wang JM, Zhang N. Load-dependent observer design for active suspension systems. International Journal of Vehicle Design, 68(1-3): 162-179, 2015.

[47] Xu F, Tan JB, Wang XQ, et al. Mixed active/passive robust fault detection and isolation using set-theoretic unknown input observers. IEEE Transactions on Automation Science and Engineering, 15(2): 863-871, 2018.

[48] Li SQ, Li J, Mo YP. Piezoelectric multimode vibration control for stiffened plate using ADRC-based acceleration. IEEE Transactions on Industrial Electronics, 61(12): 6892-6902, 2014.

[49] Li SQ, Li J, Mo YP, et al. Composite multi-modal vibration control for a stiffened plate using non-collocated acceleration sensor and piezoelectric actuator. Smart Materials and Structures, 23(1): 015006, 2014.

[50]Zheng QL, Richter H, Gao ZQ. Active disturbance rejection control for piezoelectric beam. Asian Journal of Control, 16(6): 1612-1622, 2014.

[51]Baz A. Active acoustic metamaterial with tunable effective density using a disturbance rejection controller. Journal of Applied Physics, 125(7): 074503, 2019.

[52] Nie ZY, Ma YJ, Liu RJ, et al. Improved disturbance rejection control for piezoelectric actuators based on combination of ESO and Q-filter. Electronics Letters, 54(14): 872-874, 2018.

[53] Mohanapriya S, Sakthivel R, Kwon OM, et al. Disturbance rejection for singular markovian jump systems with time-varying delay and nonlinear uncertainties. Nonlinear Analysis-Hybrid System, 33: 130-142, 2019.

[54] Chen S, Xue WC, Huang Y. Analytical design of active disturbance rejection control for nonlinear uncertain system with delay. Control Engineering Practice, 84: 323-336, 2019.

[55] Chen S, Xue WC, Zhong S, et al. On comparison of modified ADRC for nonlinear uncertain system with time delay. Science China-Information Sciences, 61(7): 70223, 2018.

[56] Castaneda LA, Luviano-Juarez A, Ochoa-Ortega G, et al. Tracking control of uncertain time delay 
system: An ADRC approach. Control Engineering Practice, 78: 97-104, 2018.

[57]Ben Amara F, Kabamba PT, Ulsoy AG. Adaptive sinusoidal disturbance rejection in linear discrete-time systems-part I: Theory. Journal of Dynamic Systems Measurement and Control-Transactions of The ASME, 121(4): 648-654, 1999. 